\title{
Grand challenges in systems physiology
}

\section{Hiroaki Kitano $0^{1,2,3 *}$}

1 The Systems Biology Institute, Tokyo, Japan

2 Sony Computer Science Laboratories, Inc. Tokyo, Japan

${ }^{3}$ Okinawa Institute of Science and Technology, Okinawa, Japan

*Correspondence: kitano@sbi.jp

Systems physiology is an integrated discipline. It combines experimental, computational, and theoretical studies to advance our understanding of the physiology of human and other living creatures. In other words, systems physiology is systems biology with a physiology (i.e., functionally)centered view. Understanding the principle behind the system is one of the fundamental challenges in systems physiology and systems biology. One can not make the use of sophisticated computational models or arrays of biological data to deepen our understanding of biological function without in-depth insights into the systems as a whole. For example, robustness and its trade-offs have been proposed as a fundamental principles (Kitano, 2004, 2007). This view, although still speculative, provides a framework for the conceptualization of data and observed phenomena. Identification of a series of such principles and their relationships can enrich our understanding of biological systems. The beauty of a good theory is that it reshapes our view of the world, so that the same data and phenomena may be re-interpreted in the light of the introduced concepts. Such transformation of our conceptualization often leads to true advances in science.

While such theoretical and explorative research are expected, it is also important to consolidate various efforts to achieve high impact objectives; these efforts are often referred as "Grand Challenges." Defining grand challenges provide an effective approach that both illuminates unresolved issues and helps focus research effort on these problems and thereby advances the stateof-the-art in systems physiology. It is most effective when used for engineering-oriented projects where progress can be made by the effective coordination of research and development programs along with a series of technological innovations, rather than merely waiting for serendipitous explorations. While basic scientific explorations are still indispensable and much needed in this field, it is also true that coordinated efforts on relatively well-defined missions can dramatically change the way we do science and apply it to medical practice. In this article, I attempt to define a series of grand challenges that are interlinked and designed to accomplish the ultimate goal of creating an integrated understanding and platform for human healthcare services, biomedical research, and drug discovery.

The grand challenge is to create highly accurate and broad coverage computational model of organisms that are backed up by well-controlled high precision experimental data. In practice, the true challenge is not only to build such a model, but also to establish a system of technologies that enable us to build these models cost-effectively, because these models must match genetic and epigenetic diversity. With this technology, both virtual human and virtual mouse models shall be developed. In addition, models of specific cell lines shall be developed. This set of models shall be consistent with a set of cells and organisms used for drug discovery and biomedical research. The reality of the drug discovery pipeline is that it uses cell lines and animal models before moving into clinical trial. Thus, it is important that not only human models, but also mouse and cell line models are developed with an equal level of quality. Accomplishment of this grand challenge will enable us to use computational models and associated experimental verification systems to understand disease mechanisms, and to predict drug efficacy, side effects, and therapeutic strategy outcomes. At a workshop held in Tokyo in February 2008, a group of researchers agreed to initiate a project to create a "virtual human" in next 30 years (Jones, 2008). They also announced the Tokyo Declaration that reads in part as follows: "Recent advances in Systems Biology indicate that the time is now ripe to initiate a grand challenge project to create over the next 30 years a comprehensive, molecules-based, multi-scale, computational model of the human ('the virtual human'), capable of simulating and predicting, with a reasonable degree of accuracy, the consequences of most of the perturbations that are relevant to healthcare."'

Although creation of a virtual human (a comprehensive computational model of human being) has been the subject of much discussion in variety of conferences and workshops, the real implications and difficulties with the model need to be readdressed. There is no doubt that simulation, if properly used, can be a powerful tool for scientific and engineering research. Modern aircrafts cannot be developed without help of computational fluid dynamics (CFD). CFD is one of the most successful computational approaches used in the engineering design process.

There are three major reasons why CFD is now widely accepted. First, the Navier-Storkes equation has been well established to provide a computational basis for fluid dynamics with reasonable accuracy. While there are yet unresolved issues on how to compute tabular flows accurately, the Navier-Storkes equation provides an acceptable practical solution for most needs. Second, many CFD results are compared and calibrated against wind-tunnel experiments that are highly controlled and extensively monitored. Due to the existence of the wind-tunnel, CFD models can be improved for their accuracy and reliability of predictions. In wind tunnels, air flow speed, temperature, and other parameters can be adjusted within a very small error margin, for example within $0.01 \%$ error margin. Third, decades of effort have been spent on improving CFD and related fluid dynamics research. Thus, the current status of CFD is a result of decades of effort.

For computer simulation and analysis in biology to parallel the success of CFD, it must establish a fundamental computing paradigm comparable to the Navier-Storkes equation, to create a wind-tunnel equivalent for biological

${ }^{1}$ http://www.systems-biology.org/ myukiko/FCSB2008/doku.php?id=workshop:statement 
experiments, and to maintain a constant focus on these problems for decades. Of course, the biological system is much more heterogeneous and complex than fluids, but a set of basic equations must be established so that fundamental principles behind the computations point in the right direction. It is essential that both interaction networks and the physical structures are modeled together so that the resulting model provides an improved reality, particularly for high-resolution modeling of complex mammalian cells. Second, highly controlled and high-precision experimental systems that will serve as the "wind-tunnel" in biology are essential. Microfluidics and other emerging technologies may provide us with experimental paradigms that have remarkably high precision (Balagadde et al., 2005).

Even if technologies can be developed, their full potential can not be reached unless they are used properly. There are, at least, two issues that must be carefully examined in order to make the best use of a computational simulation. First, the purpose of simulation has to be well defined, and model has to be constructed to maximize the purpose of the simulation. This affects the choice of modeling technique, levels of abstractions, the scope of modeling, and parameters to be varied. Second, the simulation needs to be well placed in the context of the whole analysis procedure. In most cases, simulation is not the only methods of analysis. Thus, the part of analysis that uses numerical simulation and the other parts that use non-simulation methods must be well coordinated in order to maximize overall success of the analysis activity. An example from racing car design illustrates these issues. CFD is extensively used in Formula-1 car design in order to obtain optimal aerodynamics (Ziemelis and Wenz, 2004); that is, a higher downward force coupled with a lower drag. Particular interest has been placed on effects of various aerodynamics components such as front wings, rear wings, and ground effects. However, the complicated interference between front wings, suspension members, wheels, and break air intake ducts must also be investigated. Combustion in engine is the other issue where simulation studies are often used, but simulated separately from CFD model. This exemplifies the practice of proper focus and abstraction. Thus, one can infer from this example that attempts to create a computational model of the human being without defining the model's use cases and the expected insights to be gained from the model would be economically inefficient and unlikely to be successful.

It should also be noted that CFD is not the only tool used for aerodynamics design. F-1 racing cars are initially designed using CFD (in silico), then further investigated using wind tunnel (in physico), followed by actual run at the test course (in vitro) before being deployed in actual races (in vivo). CFD in this case is used for initial search of candidate designs that are subject of further investigation and modification based upon results obtained from wind tunnel testing. This sequence of computational design followed by physical testing (experimentation) is the key for success in engineering design. It is highly likely the same would be true for biology. If so, a series of corresponding experimental platforms and methodologies may need to be developed to make the best use of the results obtained from the computational modeling approach.

Looking at the modeling platform, there are three major issues: scaling, sharing, and merging of biological models. The scaling problem, in turn, has three aspects: problem scaling, layer scaling, and scope scaling. Problem scaling means that the approach or computing framework enable models to get larger and larger to cover a substantial part of the organism. Developing a largescale model is beyond the scope and capability of a single laboratory, and, in fact, may not even be possible within a national framework. It is critically important to establish an international collaborative framework to provide the infrastructure necessary to supports these activities in order to develop large scale models. This issue is directly related to the issue of sharing and merging of models. This requires installation of platform that fosters a global initiative. For example, there must be a mechanism by which the multiple models developed by different research groups can be combined into a single consistent model. Of course, an underlying assumption is that the models can be shared, requires well-informed communication within the community and the establishment of standards for models as seen in SBML (Hucka et al., 2003) ${ }^{2}$ and SBGN (Le Novere et al., 2009)3.

\section{${ }^{2} \mathrm{http} / / /$ www.sbml.org/}

3http://www.sbgn.org/
Layer scaling means that the model can incorporate multiple layers of description from the sub-cellular, cellular, and tissue level to the whole organism and assembly of organisms. This is a non-trivial issue because each layer may have different modalities of operation and a suitable way to represent these layers into models in a consistent and integrated manner is yet to be understood.

Finally, scope scalability can be defined as the capability of modeling approach to allow for an integrated treatment of both interactions between the layers and the physical structures (Kitano, 2006). While many models often used in systems biology focus on molecular interactions and gene regulatory networks, they often neglect the important structures and dynamics of intracellular and intercellular systems as well as the whole body. This is especially the case for physiological studies. For example, models that combine cytoskeleton dynamics, hence cell deformation and movements, with molecular and genetic interactions are at best rare, but more typically totally lacking.

It must be emphasized that one must first clearly establish what scientific questions are to be answered by using computational approach before the model of the biology system is developed. While this criterion has been already stated, it is so critical for the success but all too frequently forgotten during the course of model development that I shall repeat the point again. Mere attempts to create computational models that behave like an actual cell and organisms does not in themselves constitute a good scientific practice. It must be remembered what simulation and modeling represent is an abstraction of the actual phenomena. Without first carefully framing the scientific questions, a proper determination of the right level of abstraction and the scope of the model to be created is not possible. This is also the case in CFD. CFD as used in racing car design has a clear and an explicit optimization goal, namely to maximize the downward force while minimizing drag. The problem for biological simulations is that the information to be discovered by the simulation is much more complex than needed for racing car design. However, the questions must be a well-defined one in order to make the best 
use of computational machinery. With the right question and framing of problem, the model can become the starting point for a broad range of applications.

The discussion as presented so far outlines a new set of problems and challenges that has not been common in traditional biomedical sciences. This fact may have implications on how scientific communications, including journals, need to be organized and directed. For example, models and other resources that are gaining more importance have not been properly credited. There needs to be mechanisms by which proper credit can be assigned to the large groups of international experts who contribute to the incremental improvement of existing models and other knowledge resources. The proper consolidation of knowledge is as equally important for scientific advancement as are novel discoveries because the simple assembly of isolated knowledge, regardless of originality of discovery itself, does not enable us to achieve the grand challenge.

In order to resolve this issue, this journal attempts to provide a forum for the publication of modeling and mapping studies, results that have often been difficult to publish. The development of precision models, molecular interactions maps, and other knowledge-intensive resources are critical for the advancement of systems physiology and systems biology. In the past, the value of submissions describing these results have not been fully appreciated due to the assumption that these studies fail to provide novel insights despite the fact that important insight is often gained by efficient use of these models and maps. Just like an engineer who can design and build a great car without being a great driver, those who develop precision models and maps can provide functional insights that others can experimentally confirm or refute. In a similar fashion, being a great driver does not means that one can design and build a great car. Thus, someone who can gain insights from the models and maps may not be the one who can actually develop the resources used in the model. Model and map construction are engineering and infrastructure work that requires specific skills and dedication that provide resources essential for promoting systems physiology and such contributions need to be properly credited. A major goal of this journal is to establish an innovative forum of scientific exchange in the new area of web-based scientific activity.

A grand challenge for systems physiology entails this exciting objective. We need a series of innovations, discoveries, collaborative efforts, and dedications to accomplish it. The impact will be massive.

\section{REFERENCES}

Balagadde, F. K., You, L., Hansen, C. L., Arnold, F. H., and Quake, S. R. (2005). Long-term monitoring of bacteria undergoing programmed population control in a microchemostat. Science 309, 137-140.

Hucka, M., Finney, A., Sauro, H. M., Bolouri, H., Doyle, J. C., Kitano, H., Arkin, A. P., Bornstein, B. J., Bray, D., Cornish-Bowden, A., Cuellar, A. A., Dronov, S., Gilles, E. D., Ginkel, M., Gor, V., Goryanin, I. I., Hedley,
W. J., Hodgman, T. C., Hofmeyr, J.-H., Hunter, P. J., Juty, N. S., Kasberger, J. L., Kremling, A., Kummer, U., Le Novere, N., Loew, L. M., Lucio, D., Mendes, P., Minch, E., Mjolsness, E. D., Nakayama, Y., Nelson, M. R., Nielsen, P. F., Sakurada, T., Schaff, J. C., Shapiro, B. E., Shimizu, T.S., Spence, H. D., Stelling, J., Takahashi, K., Tomita, M., Wagner, J., and Wang, J. (2003). The systems biology markup language (SBML): a medium for representation and exchange of biochemical network models. Bioinformatics 19, 524-531.

Jones, D. (2008). All Systems Go. Nat. Rev. Drug Discov. 7, 128-129.

Kitano, H. (2004). Biological robustness. Nat. Rev. Genet. $5,826-837$.

Kitano, H. (2006). Computational cellular dynamics: a network-physics integral. Nat. Rev. Mol. Cell Biol. 7, 163.

Kitano, H. (2007). Towards a theory of biological robustness. Mol. Syst. Biol. 3, 137.

Le Novere, N., Hucka, M., Mi, H., Moodie, S., Schreiber, F., Sorokin, A., Demir, E., Wegner, K., Aladjem, M. I., Wimalaratne, S. M., Bergman, F. T., Gauges, R., Ghazal, P., Kawaji, H., Li, L., Matsuoka,Y., Villéger, A., Boyd, S.E., Calzone, L., Courtot, M., Dogrusoz, U., Freeman, T. C., Funahashi,A., Ghosh, S., Jouraku, A., Kim, S., Kolpakov, F., Luna, A., Sahle, S., Schmidt, E., Watterson, S., Wu, G., Goryanin, I., Kell, D. B., Sander, C., Sauro, H., Snoep, J. L., Kohn, K., and Kitano, H. (2009). The systems biology graphical notation. Nat. Biotechnol. 27, 735-741.

Ziemelis, K., and Wenz, C. (2004). Formula 1 racing: Science in the FAST LANE. Nature 431, 736-738.

Received: 28 March 2010; accepted: 31 March 2010; published online: 07 May 2010.

Citation: Kitano H. (2010) Grand challenges in systems physiology. Front. Physio. 1:3. doi: 10.3389/fphys.2010.00003

This article was submitted to Frontiers in Systems Physiology, a specialty of Frontiers in Physiology.

Copyright (c) 2010 Kitano. This is an open-access article subject to an exclusive license agreement between the authors and the Frontiers Research Foundation, which permits unrestricted use, distribution, and reproduction in any medium, provided the original authors and source are credited. 